\title{
MAPS OF THE INTERVAL WITH EVERY POINT CHAIN RECURRENT
}

\author{
LOUIS BLOCK AND ETHAN M. COVEN
}

\begin{abstract}
We show that if $f$ is a continuous map of a compact interval to itself and every point is chain recurrent, then either $f^{2}$ is the identity map or $f^{2}$ is turbulent.
\end{abstract}

In this paper, we show that the dynamics of maps of the interval for which every point is chain recurrent must be either completely trivial or quite complex. Specifically, we prove the following

THEOREM. Let $f$ be a continuous map of a compact interval to itself. If every point is chain recurrent, then either $f^{2}$ is the identity map or $f^{2}$ is turbulent.

In the latter case, $f$ has periodic points of all even periods and topological entropy at least $\frac{1}{2} \log 2$.

Note that the hypothesis that every point is chain recurrent is satisfied if the map is transitive or if the set of periodic points is dense (equivalently, if every point is nonwandering). In particular, the theorem extends results $[\mathbf{3}, \mathbf{1}]$ which show that if $f$ is transitive, then $f^{2}$ is turbulent, and if the set of periodic points is dense, then either $f^{2}$ is the identity map or $f^{2}$ is turbulent.

We recall the definitions and some basic facts about chain recurrence and turbulence. Let $g$ be a continuous map of a compact metric space $X$, with metric $d$, to itself. For $x, y \in X$ and $\varepsilon>0$, an $\varepsilon$-chain from $x$ to $y$ is a finite sequence $x=x_{0}, \ldots, x_{n}=y$ where $n>0$ and $d\left(g\left(x_{i}\right), x_{i+1}\right)<\varepsilon$ for $0 \leq i \leq n-1$. We say that $x$ is chain recurrent (under $g$ ) if for every $\varepsilon>0$, there is an $\varepsilon$-chain from $x$ to $x$. It follows from the definitions that every nonwandering point is chain recurrent and that if every point is chain recurrent, then $g$ maps $X$ onto $X$. Furthermore, for every $n>0, x$ is chain recurrent under under $g$ if and only if it is chain recurrent under $g^{n}$. (Although it is not directly relevant to this paper, it can be shown, using results from [4], that if $X$ is connected and every point is chain recurrent, then $g$ is chain transitive, i.e., for every $x, y \in X$ and every $\varepsilon>0$, there is an $\varepsilon$-chain from $x$ to $y$.)

Lemma 1 follows from $[4$, Theorem A].

LEMMA 1. If $X$ is connected and every point is chain recurrent, then there is no nonempty open set $U \neq X$ such that $g(\bar{U}) \subseteq U$.

Our main concern in this paper is with maps of the interval. A continuous map $f$ of a compact interval $I$ to itself is called turbulent $[2]$ if there are nondegenerate

Received by the editors July 15, 1985.

1980 Mathematics Subject Classification (1985 Revision). Primary 54H20.

This work was done while the first author was a J. H. Van Vleck Visiting Professor at Wesleyan University. 
closed intervals $J, K \subseteq I$ with at most one point in common, such that $f(J) \cap$ $f(K) \supseteq J \cup K$. The following result can be proved using standard methods (see $[\mathbf{5}])$.

LEMMA $2[\mathbf{2}]$. If $f$ is turbulent, then

(1) $f$ has more than one fixed point,

(2) $f$ has periodic points of all periods,

(3) $h(f) \geq \log 2$, where $h$ denotes topological entropy.

LEMMA 3. If $f$ is onto, then $f^{2}$ has more than one fixed point.

PROOF. If $f$ has more than one fixed point, there is nothing to prove. So suppose that $f$ has a unique fixed point $p$. We may assume that the ambient interval is $[0,1]$. Then $p \neq 0,1$ and $f(x)>x$ for all $x \in[0, p)$ and $f(x)<x$ for all $x \in(p, 1]$. Since $f$ is onto, $f[0, p] \supseteq[p, 1]$ and $f[p, 1] \supseteq[0, p]$. Hence $f^{2}[0, p] \supseteq[0, p]$ and so there exists $a \in[0, p)$ such that $f^{2}(a)=0$. Then $f^{2}$ has a fixed point in $[0, a]$.

The heart of the proof of the theorem is the following lemma, which extends [3, Lemma 3.3].

LEMMA 4. If $f$ has every point chain recurrent, then exactly one of the following conditions holds.

(1) $f$ is the identity map.

(2) $f$ has exactly one fixed point.

(3) $f$ is turbulent.

PROOF. Again we may assume that the ambient interval is $[0,1]$. Suppose that $f$ is not the identity map and that $f$ has more than one fixed point. We show that $f$ is turbulent. By Lemma 1 , there is no nonempty open set $U \neq[0,1]$ such that $f(\bar{U}) \subseteq U$.

Case 1. There are fixed points $a<b$ such that $f$ has no fixed points in $(a, b)$.

Without loss of generality, we may assume that $f(x)<x$ for every $x \in(a, b)$. Then $a \neq 0$, for otherwise $U=\left[0, b^{\prime}\right)$ satisfies $f(\bar{U}) \subseteq U$ for any $b^{\prime} \in(a, b)$.

Let $c$ be the largest point in $[0, a)$ such that $f(c)=b$. (If no such $c$ exists, then there exists $b^{\prime} \in(a, b)$ such that $f(x)<b^{\prime}$ for all $x \in[0, a)$. But then $U=\left[0, b^{\prime}\right)$ satisfies $f(\bar{U}) \subseteq U$.) Let $d$ be any point in $(c, b)$ such that $f(d)=c$. (Again if no such $d$ exists, then there exists $c^{\prime} \in(c, a)$ such that $f(x)>c^{\prime}$ for all $x \in[c, b)$. But then there exists $b^{\prime} \in(a, b)$ such that $U=\left(c^{\prime}, b^{\prime}\right)$ satisfies $f(\bar{U}) \subseteq U$.) Then $J=[c, d]$ and $K=[d, b]$ show that $f$ is turbulent.

Case 2. For every pair of fixed points $a<b$, there is a fixed point in $(a, b)$.

Then the set of fixed points is a nondegenerate closed interval $[p, q]$. Since $f$ is onto, $p \neq 0$ and $q \neq 1$. Furthermore, $f(x)>x$ for all $x \in[0, p)$ and $f(x)<x$ for all $x \in(q, 1]$. There are connected neighborhoods $V$ of $p$ and $W$ of $q$ such that $f(x)<q$ for all $x \in V$ and $f(x)>p$ for all $x \in W$. Let $p^{\prime}<p$ be in $V$ and $q^{\prime}>q$ be in $W$. Then $U=\left(p^{\prime}, q^{\prime}\right)$ satisfies $f(\bar{U}) \subseteq U$.

Therefore Case 2 is impossible and the proof is complete.

Now to prove the theorem, observe that every point is chain recurrent under $f^{2}$. Then by Lemmas 3 and 4 (the latter applied to $f^{2}$ ), either $f^{2}$ is the identity map of $f^{2}$ is turbulent. 
A nonempty, proper closed subset $K$ of $X$ is called an attractor for $g: X \rightarrow X$ if there is an open set $U$ containing $K$ such that

$$
K=\bigcap_{n \geq 0} \bigcup_{m \geq n} g^{m}(U) .
$$

By $[4$, Corollary $\mathrm{C}]$, if $X$ is connected and locally connected, and if some point of $X$ is not chain recurrent, then some power of $g$ has a connected attractor. Therefore we have the following corollary.

COROLlaRY 1. For any map $f$ of the interval, at least one of the following conditions holds.

(1) $f^{2}$ is the identity map.

(2) $f^{2}$ is turbulent.

(3) There is a proper closed, possibly degenerate, subinterval which is an attractor for some power of $f$.

The second corollary is an immediate consequence of theorem, Lemma 2, and the fact that $h\left(f^{2}\right)=2 h(f)$. It had previously been shown [3, Corollary $3.5 ; 6$, Lemma 1.2] that the conclusions of this corollary hold if $f$ is transitive.

COROLlaRY 2. With $f$ as in the theorem, if $f^{2}$ is not the identity map, then $f$ has periodic points of all even periods and $h(f) \geq \frac{1}{2} \log 2$.

The map $f:[0,3] \rightarrow[0,3]$ which satisfies $f(0)=2, f(1)=3, f(2)=2, f(3)=0$, and which is linear between these points, shows that both bounds are sharp.

We close with an example of a map of the interval for which every point is chain recurrent, but the set of nonwandering points is nowhere dense. Let $f:[0,3] \rightarrow[0,3]$ be defined as follows. Let $f(x)=\sqrt{x}$ for $x \in[0,1], f(2)=3, f(3)=0$, and let $f$ be linear on $[1,2]$ and $[2,3]$. It is easy to check that every point is chain recurrent and that the set of nonwandering points is $\{0\} \cup C$, where $C$ is a Cantor set in $[1,8 / 3]$. Furthermore, $f \mid C$ is topologically conjugate to the full one-sided shift on two symbols.

\section{REFERENCES}

1. M. Barge and J. Martin, Dense periodicity on the interval, Proc. Amer. Math. Soc. 94 (1985), 731-735.

2. L. Block and W. A. Coppel, Stratification of continuous maps of an interval, Trans. Amer. Math. Soc. 297 (1986), 587-604.

3. L. Block and E. Coven, Topological conjugacy and transitivity for a class of pieceurise monotone maps of the interval, Trans. Amer. Math. Soc. (to appear).

4. L. Block and J. Franke, The chain recurrent set, attractors, and explosions, Ergodic Theory Dynamical Systems 5 (1985), 321-327.

5. L. Block, J. Guckenheimer, M. Misiurewicz, and L.-S. Young, Periodic points and topological entropy of one-dimensional maps, Global Theory of Dynamical Systems (Proc. Internat. Conf., Northwestern Univ., Evanston, Ill., 1979), Lecture Notes in Math., Vol. 819, Springer, Berlin, 1980, pp. 18-34.

6. A. Blokh, On sensitive mappings of an interval, Russian Math. Surveys 37 (1982), 203-204. 32611

Department of MAThematics, University of Florida, Gainesville, Florida

Department of MAThematics, Wesleyan University, Middletown, ConneCtiCUT 06457 